\title{
Effects of Attentional Focus on Walking Stability in Elderly
}

Jonathan L. A. de Melker Worms ${ }^{1,3 *}$, John F. Stins ${ }^{1}$, Erwin E. H. van Wegen ${ }^{2}$, Sabine M. P. Verschueren ${ }^{4}$, Peter J. Beek ${ }^{1}$, Ian D. Loram ${ }^{3}$

${ }^{1}$ Department of Human Movement Sciences, Faculty of Behavioural and Movement Sciences, Vrije Universiteit Amsterdam, MOVE Research Institute Amsterdam, The Netherlands.

${ }^{2}$ Department of Rehabilitation Medicine, VU University Medical Center, MOVE Research Institute Amsterdam, The Netherlands.

${ }^{3}$ Cognitive Motor Function research group, School of Healthcare Science, Manchester Metropolitan University, Manchester, United Kingdom.

4 Research group of musculoskeletal rehabilitation, Department of rehabilitation, Katholieke Universiteit, Leuven, Belgium.

\section{* Corresponding author}

Contact:

Cognitive Motor Function research group, School of Healthcare Science, Manchester Metropolitan University, John Dalton Building, Oxford Road, Manchester M1 5GD, United Kingdom.

Email: j.demelkerworms@gmail.com

\section{Co-author email addresses}

John F. Stins:

j.f.stins@vu.nl

Erwin van Wegen:

E.vanWegen@vumc.nl

Sabine M.P. Verschueren:

Peter J. Beek:

sabine.verschueren@faber.kuleuven.be

Ian D. Loram:

p.j.beek@vu.nl

i.loram@mmu.ac.uk

\section{Acknowledgement}

This work is part of the MOVE-AGE joint doctorate programme, which is funded by the European Commission as part of the Erasmus Mundus programme with grant number: 2014 - 0691 / 001 - 001 - EMJD. We would like to thank Mary Kraus, Aimée van Nieuwenhuijzen and Daniëlle Bouman for their assistance during the experiment. 


\begin{abstract}
Introduction: Falling is one of the main causes of injury-related hospitalisation and mortality in older adults. We investigated the effects of internal vs. external focus of attention and fall history on walking stability in healthy older adults. Method: Walking stability was assessed by applying random unilateral decelerations on a splitbelt treadmill and analysing the resulting balance recovery movements. The internal focus instruction was: Concentrate on the movement of your legs, whereas the external focus instruction was: Concentrate on the movement of the treadmill. In both conditions participants were asked to look ahead at a screen. Outcome measures were coefficient of variation of step length and step width, and characteristics of the centre of mass velocity time-series as analysed using statistical parametric mapping. Fall history was assessed using a questionnaire. Results: After each perturbation participants required two to three strides to regain a normal gait pattern, as determined by the centre of mass velocity response. No effects were found of internal and external focus of attention instructions and fall history on any of the outcome measures. Discussion: We conclude that, compared to an internal focus of attention instruction, external focus to the walking surface does not lead to improved balance recovery responses to gait perturbations in the elderly.
\end{abstract}

\title{
Keywords
}

Gait; Elderly; Falls; Gait Perturbations; Attention; Motion capture 


\section{Introduction}

Falling is one of the main causes of injury-related hospitalisation and mortality in elderly [1]. Fall risk and decline of balance performance in the elderly are not solely related to physical degeneration since psychological factors like attentional focus may be involved as well. Individuals with increased fall risk have heightened conscious attention to their own movements, which otherwise would be more automated and require less attentional control [2-4].

In literature a distinction is made between an external and internal focus of attention. Wulf and Prinz [5] described an internal focus as directing the performers' attention to movement of their own body, e.g. towards movements of their feet while standing on an unstable balance board [6-8]. In contrast, an external focus was described as directing attention to the effect of the movement in the environment, e.g. movement of a balance board one is standing on [6-8]. In some tasks, however, the goal is not to move or act upon an external object, but to control movement of the body itself relative to the environment. In that case external focus comprises directing attention to the surface on which force is exerted by the human performer and which is relevant to successful motor performance, e.g. the ground one is standing on in gymnastics [2, $9,10]$.

An external focus of attention has generally been found to result in superior motor performance than an internal focus of attention [2]. According to the constrained action hypothesis [7], an internal focus constrains or interferes with automatic control processes that would normally regulate movement, whereas an external focus facilitates efficient task-performance by allowing the motor system to more naturally organize itself $[5,7,11-13]$. The detrimental effect of an internal focus is not confined to motor performance, but extends to motor learning [2]. For example, balance performance increased faster in older adults learning a new balance task with an external focus rather than an internal focus [8]. Fall-prone elderly might adopt a more internally directed focus as a protective strategy, especially when walking stability is challenged, resulting in reduced walking performance. Furthermore, physical therapists are inclined to employ more internal than external focus instructions and feedback in gait re-education, which might attenuate motor learning [14]. 
However, to our knowledge it has never been investigated whether attentional focus instructions alone can alter gait performance in the elderly, and whether their effects are modulated by fall history. In this study we investigated the combined effects of attentional focus and fall history on walking stability in healthy elderly.

\section{Aims and hypotheses}

Based on previous findings, we hypothesised that an external focus temporarily leads to a more stable perturbed walking pattern than an internal focus of attention. To assess walking stability we applied unilateral mechanical perturbations on a split-belt treadmill $[15,16]$, and examined the balance recovery process. Such perturbations are experienced as a forward slip of the foot, e.g., when walking on a slippery surface. Fall history and decreased walking stability are associated with increased variability [17]. We therefore operationalised our main hypothesis in two more detailed expectations, namely that compared to an internal focus, an external focus would lead to (1) decreased variability of perturbed step length and step width and (2) faster recovery to a stable walking pattern as determined on the basis of centre of mass (COM) velocity profiles. Additionally, we examined whether the effect of attentional focus on walking stability is dependent on the fall history of the participants.

\section{Method}

\section{Participants}

Twenty-eight healthy older adults ( 8 males, 20 females) aged 65 or above, who were able to walk independently for 10 minutes, were recruited. Their average age was $69.3 \pm 3.7$ years (range: $65-78$ ). A Dutch version of the Mini-Mental State Examination (MMSE) was used to determine the cognitive status of the participants. Participants with a MMSE score below 25/30 were excluded. The study received approval from the local ethical committee and participants gave written informed consent prior to their participation.

\section{Material}

Participants walked on the Gait Real-time Analysis Interactive Lab (GRAIL) system (Motekforce Link b.v., Amsterdam, The Netherlands), which consists of a split-belt treadmill in combination with a Virtual Environment (VE) projected on a $180^{\circ}$ semi- 
cylindrical screen (Fig. 1). This system allowed us to perturb gait by applying transient unilateral treadmill decelerations. The VE in this experiment comprised a straight road, surrounded by forest and mountains, providing realistic optical flow while walking. Motekforce Link's D-flow software was used to control the system. Ten high-resolution infra-red cameras (Vicon, Oxford, UK) and the Human Body Model (HBM, Motekforce Link) full-body marker set were used to capture kinematic data at $100 \mathrm{~Hz}$ using 47 passive retroreflective markers [18]. A safety harness system suspended overhead prevented participants from falling without weight support.

\section{Fall history}

Participants filled out a fall history questionnaire before the experiment. A fall was defined as an event in which a person unintentionally comes to rest on the ground or other lower levels [19]. Participants who had experienced a fall within 12 months before the experiment were labelled as fallers; the other participants were labelled as non-fallers. Falls that resulted from loss of consciousness or acute paralysis caused by stroke, epileptic attacks or violence were excluded.

\section{Procedure}

Participants were instructed to always look ahead at the screen and familiarised with treadmill walking at a speed of $1 \mathrm{~m} / \mathrm{s}$ and with the perturbations. In all trials this fixed speed was used to have a common reference for examining the perturbation responses. Perturbations consisted of brief unilateral decelerations of the split-belt treadmill on the participant's dominant leg side only, and occurred at random intervals between 10 and 20 seconds. Unilateral treadmill decelerations initiated at toe-off of the dominant foot. At the following heel strike of the same foot the belt was decelerated to $0 \mathrm{~m} / \mathrm{s}$. This resulted in a motor response resembling a forward foot slip. At the next heel strike of the same foot, the belt had regained the original velocity of 1 $\mathrm{m} / \mathrm{s}$.

The experiment comprised two perturbed gait trials of five minutes per participant, one for the internal and one for the external focus condition in counterbalanced order. In each condition 20 perturbations were applied. In the internal focus condition participants received the following verbal instruction: "Look ahead at the screen and concentrate on the movement of your legs", whereas in the external focus condition 
they received this instruction: "Look ahead at the screen and concentrate on the movement of the treadmill". Instructions were repeated every 30 seconds during the trials using a speaker system. As this experiment was part of a multi-experiment protocol, participants had already walked $1 \mathrm{~m} / \mathrm{s}$ for 20 minutes at the start of this particular experiment.

\section{Data analysis: Step length \& step width}

The mean step length and step width of the first recovery step following each perturbed heel strike was determined based on heel and toe marker positions. Furthermore the coefficients of variation $(C V)$ of step length and step width were calculated for each participant (standard deviation as a percentage of the mean) [20].

Step length and step width data were analysed using Matlab (version R2014a, The MathWorks, Inc., Natick, MA, USA).

\section{Data analysis: Normalised Euclidean distance (D)}

The normalised Euclidean distance was calculated as a measure of deviation from a participant's normal gait pattern. From the walking episodes, participants' body COM was calculated using Visual 3D (v5.02.07, C-Motion Inc., Germantown, USA). The velocity of the X-, Y-and Z-time-series of the COM was calculated through differentiation using a 4th order Savitsky-Golay filter with a temporal window of 90 ms [21]. These time-series were then normalised using spline interpolation, such that every stride consisted of 100 samples. The COM velocity data between $4 \mathrm{~s}$ after each perturbation up until the next perturbation were classified as unperturbed walking (UW) bouts. The UW bouts of these time-series were combined to create an average limit cycle for each subject and condition (internal vs. external focus). This limit cycle represents the average $\mathrm{COM}$ behaviour at each percentage of an unperturbed stride in that condition. Furthermore, for each percentage in this limit cycle, the standard deviation in unperturbed walking $\left(v_{\mathrm{UW}}\right)$ was calculated for each dimension. Walking bouts ranging from the first stride before each perturbation until the fourth stride after the perturbation were classified as perturbed walking (PW) bouts. The normalised Euclidean distances $(D)$ of the COM velocity time-series between PW bouts and the 
average limit cycle (UW) were then calculated as described by Bruijn et al. (2010) [16], see equation (1).

$$
D(k \times 100+i)_{\substack{k=0: n-1 \\ i=1: 100}}=\sqrt{\sum_{d=1}^{3}\left(\left(\mathrm{UW}(i)_{d}-\mathrm{PW}(\mathrm{k} \times 100+i)_{d}\right) / v_{\mathrm{UW}}(i)_{d}\right)^{2}}
$$

$D(k \times 100+i)$ is the normalised distance (in standard deviations) for $i \%$ of stride $k+1$ (with $n$ representing the maximum number of strides in $\mathrm{PW}$ ); $d$ is the spatial dimension number, $\mathrm{UW}$ is the limit cycle, $\mathrm{PW}$ is the state of the perturbed walking trial, and $v_{\mathrm{UW}}$ is the variability of the limit cycle. The COM data were analysed using Matlab.

\section{Step length and step width statistics}

A $2 \times 2$ mixed ANOVA including effect sizes (partial $\eta^{2}$ ) and Bayes factors were calculated to test whether participant means of step length and step width were significantly different between focus conditions, between fallers and non-fallers and whether fall history interacted with attentional focus. The step width $C V$ and step length $C V$ data did not pass the Shapiro-Wilk test for normality. Therefore Wilcoxon signed-rank tests $(\mathrm{Z})$ were used to compare differences between internal and external focus conditions. Fallers and non-fallers were compared using Mann-Whitney U tests. For fall history effects within focus conditions, subsequent Mann-Whitney U tests with Šidák correction were used, while subsequent Wilcoxon signed-rank tests with Šidák corrections were used for focus condition effects within fallers and non-fallers. For all tests on $C V$ data, effect sizes (r) and Bayes factors were also calculated. Statistics of means and $C V s$ of step width and step length were calculated with IBM SPSS Statistics 20.0, except for the Bayes factors, which were calculated with the BayesFactor v0.9.12-2 package for R (bayesfactorpcl.r-forge.r-project.org; Rproject.org).

\section{Statistical Parametric Mapping (SPM)}

As our second expectation pertained to $D$ at each percentage of the post-perturbation strides, we used a validated method of time-series analysis (i.e. SPM) to test whether 
the $D$ time-series were significantly different between conditions. All SPM analyses were implemented using the open-source toolbox SPM-1D (v.M0.1, Todd Pataky 2014) [22] in Matlab R2014a. SPM regards the whole time-series as the unit of observation and is gaining ground in the analysis of kinematic time-series [23-25]. This allows time dependence to be incorporated directly in statistical testing.

A SPM two-tailed one-sample $t$-test was used separately for each focus condition to test whether $D$ was different from the relaxation distance $(\alpha=0.05)$. Additionally a SPM two-tailed paired samples $t$-test [23] was used for an internal vs. external focus comparison of $D$. The scalar output statistic, $\operatorname{SPM}\{\mathrm{t}\}$, was calculated separately at each individual time sample. To test the null hypothesis, the critical threshold was calculated at which only $\alpha \%$ (5\%) of the analysed trajectories would be expected to traverse. This threshold is based upon estimates of trajectory smoothness [26] and Random Field Theory expectations [27]. Conceptually, a SPM $t$-test is similar to the calculation and interpretation of a scalar $t$-test; if the SPM $\{\mathrm{t}\}$ trajectory crosses the critical threshold at any time sample, the null hypothesis is rejected. However, a SPM $t$-test mitigates the false positives of a scalar $t$-test and the false negatives of a Bonferroni corrected scalar t-test [27].

\section{Results}

Mean and $C V$ of step width \& step length

The mean and $C V$ of step length and step width of the first recovery step following the perturbed heel strikes is shown in Fig. 2. Inspection of the data revealed that three participants adopted a different recovery strategy than the other participants. In response to the perturbation this different strategy involved an initial abrupt backward step in both conditions, after which a normal stepping pattern was resumed.

Calculation of step length for these participants would result in negative values; therefore these three participants (one faller, two non-fallers) were excluded from the step length and step width analysis. The scatter plot in Fig. 2 shows the data for the remaining 25 participants. No significant difference was found for any of the spatiotemporal parameters between focus conditions or between fallers and nonfallers. The interaction effect between attentional focus and fall history was also not significant. Furthermore, for the main effect of focus, the Bayes factors for the $C V s$ of step width and step length were smaller than 0.33 . Therefore the odds for the null- 
hypothesis (no difference) vs. the alternative hypothesis are higher than 3 to 1 for the $C V$ variables, see Table. 1.

\section{Euclidean distances}

The averaged earth-vertical (up and down) COM position time-series during perturbed and unperturbed walking are shown for a representative participant in Fig. 3. It displays how the perturbation causes the time-series to diverge for internal and external focus conditions.

The normalised Euclidean distances $(D)$ and the corresponding SPM analysis are shown in Fig. 4. After perturbation the distance to the unperturbed walking pattern quickly increased and then gradually moved back to the relaxation distance. This relaxation distance resulted from the natural variability of unperturbed gait, i.e. UW bouts [16]. For both conditions the perturbations caused a COM velocity response that was significantly different from unperturbed walking for more than one stride after the perturbation onset.

For the internal focus condition the difference from unperturbed walking was significant from $4 \%$ of the first stride until $78 \%$ of the second stride $(178 \%)$ after perturbation onset $(\mathrm{p}<0.01)$. For the external focus condition the difference was significant from $4 \%$ to $236 \%$ ( $<<0.01)$. As the confidence intervals for the external focus condition are slightly smaller than for the internal focus condition between $178 \%$ and $236 \%$, the internal focus SPM graph falls below the threshold of significance in that time window, whereas the external focus SPM graph stays above this threshold. This difference is not caused by a difference of the mean responses between conditions, which is evidenced by the lack of a significant difference between conditions as indicated by the difference (green) graph. The origin of the difference in this time window lies in the slightly smaller between-subjects variability in the external focus condition compared to the internal focus condition, as shown by the confidence intervals. So even though the stride percentages at which these effects cease to be significant for the internal and external focus condition are 58\% apart, no significant difference between these conditions was found as shown by the SPM paired $t$-test graph (Fig. 4). 


\section{Discussion}

In this study we investigated the effect of attentional focus and fall history on walking stability as assessed by means of transient mechanical perturbations. No significant difference between internal and external focus and between fallers and non-fallers was found for means and $C V s$ of step length and step width of the first step following perturbation. This disconfirmed our first expectation that external focus during walking leads to decreased variability of perturbed step length and step width compared to internal focus in elderly. Moreover, no significant effect of attentional focus was found in the COM velocity during the first four strides following each perturbation. This disconfirmed our second expectation that external focus leads to faster recovery to a stable gait pattern in elderly than internal focus. Therefore, in contrast to previous findings [13], the beneficial effects of external vs. internal focus on motor performance do not seem to apply to balance control during walking, that is, for the instructions as used in the present study.

\section{Possible reasons for the absence of attentional effects}

When the task is to move and act upon an external object, directing one's attention to that object has been shown to produce better performance of a variety of motor tasks (e.g., far aiming, jumping and balancing tasks) than directing attention to one's own body movements [2]. Collectively, this research has indicated that an external focus is more useful to the planning and execution of goal-directed instrumental actions than an internal focus. In the present experiment the participants' goal was not to achieve a particular perceptible environmental effect but rather to maintain an upright walking pattern. They had to control the movement and location of their own body and no external focus instructions could be given in relation to a particular environmental effect. Visual information about the environment aids to determine one's location. Therefore the instruction to look ahead at the screen could have been more useful to provide information about body movement than concentrating on the movement of the legs or treadmill belt. Other studies in which the participants' task was to produce a specific bodily movement have shown mixed results. For instance, performance benefits of an external focus of attention have been found for the golf swing form [9], but not for gymnastics [10]. In stroke patients even an opposite effect has been found in that an internal focus led to better paretic leg movement performance than an external focus [28]. 
Carson and Collins [29] recently disputed the prevailing notion that an internal 'selffocus' of attention invariably results in poorer motor performance. They argued that motor learning benefits from a self-focus on the body movement as a whole rather than a partial self-focus on one of its components. In most studies investigating effects of attentional focus on motor performance, including the present study, a partial form of self-focus was used as internal focus condition [2, 5, 7, 11-13]. Therefore, future studies comparing the effects of different forms of internal focus instructions on walking performance seem required to better understand the mechanisms underlying attentional focus effects.

\section{Conclusion}

No significant difference was found between internal and external focus conditions on parameters associated with walking stability like step length $C V$, step width $C V$ and COM velocity following a brief mechanical perturbation. This might be caused by the absence of an external object to move or act upon. We therefore conclude that for elderly gait, attending to the walking surface does not lead to improved balance recovery responses to gait perturbations.

\section{Conflict of interest statement}

None declared

\section{Literature}

[1] Rubenstein LZ. Falls in older people: epidemiology, risk factors and strategies for prevention. Age and ageing. 2006;35 Suppl 2:ii37-ii41.

[2] Wulf G. Attentional focus and motor learning: a review of 15 years. Int Rev Sport Exer P. 2013;6:77-104.

[3] Wong WL, Masters RS, Maxwell JP, Abernethy AB. Reinvestment and falls in community-dwelling older adults. Neurorehabilitation and neural repair. 2008;22:410-4.

[4] Young WR, Olonilua M, Masters RS, Dimitriadis S, Mark Williams A. Examining links between anxiety, reinvestment and walking when talking by older adults during adaptive gait. Experimental brain research Experimentelle Hirnforschung Experimentation cerebrale. 2015.

[5] Wulf G, Prinz W. Directing attention to movement effects enhances learning: a review. Psychonomic bulletin \& review. 2001;8:648-60. 
[6] McNevin N, Weir P, Quinn T. Effects of attentional focus and age on

[7] McNevin NH, Shea CH, Wulf G. Increasing the distance of an external focus of attention enhances learning. Psychological research. 2003;67:22-9.

[8] Chiviacowsky S, Wulf G, Wally R. An external focus of attention enhances balance learning in older adults. Gait Posture. 2010;32:572-5.

[9] An J, Wulf G, Kim S. Increased Carry Distance and X-Factor Stretch in Golf Through an External Focus of Attention. Journal of Motor Learning and Development. 2013;1:2-11.

[10] Lawrence GR, Gottwald VM, Hardy J, Khan MA. Internal and external focus of attention in a novice form sport. Research quarterly for exercise and sport. 2011;82:431-41.

[11] Lohse KR, Sherwood DE, Healy AF. How changing the focus of attention affects performance, kinematics, and electromyography in dart throwing. Hum Movement Sci. 2010;29:542-55.

[12] Freudenheim AM, Wulf G, Madureira F, Pasetto SC, Corea UC. An External Focus of Attention Results in Greater Swimming Speed. International Journal of Sports Science \& Coaching. 2010;5:533-42.

[13] Ducharme SW, Wu WF. An external focus of attention improves stability after a perturbation during a dynamic balance task. Journal of Motor Learning and Development. 2015;3:74-90.

[14] Johnson L, Burridge JH, Demain SH. Internal and external focus of attention during gait re-education: an observational study of physical therapist practice in stroke rehabilitation. Physical therapy. 2013;93:957-66.

[15] Granacher U, Gruber M, Forderer D, Strass D, Gollhofer A. Effects of ankle fatigue on functional reflex activity during gait perturbations in young and elderly men. Gait Posture. 2010;32:107-12.

[16] Bruijn SM, Meijer OG, Beek PJ, van Dieen JH. The effects of arm swing on human gait stability. The Journal of experimental biology. 2010;213:3945-52. [17] Toebes MJ, Hoozemans MJ, Furrer R, Dekker J, van Dieen JH. Local dynamic stability and variability of gait are associated with fall history in elderly subjects. Gait Posture. 2012;36:527-31.

[18] van den Bogert AJ, Geijtenbeek T, Even-Zohar O, Steenbrink F, Hardin EC. A real-time system for biomechanical analysis of human movement and muscle function. Medical \& biological engineering \& computing. 2013;51:1069-77. [19] de Zwart AH, van der Esch M, Pijnappels MA, Hoozemans MJ, van der Leeden M, Roorda LD, et al. Falls Associated with Muscle Strength in Patients with Knee Osteoarthritis and Self-reported Knee Instability. The Journal of rheumatology. 2015;42:1218-23. [20] Abdi H. Coefficient of variation. Encyclopedia of research design. 2010:16971.

[21] Press WH, Teukolsky SA, Vetterling WT, Flannery BP. Numerical recipes in C. The art of scientific computing. Cambridge: Cambridge University Press.; 1999. [22] Pataky T. SPM-1D, v.M0.1. One-dimensional statistical parametric mapping. http://www.spm1d.org; 2014 [accessed 3-12-2014]. [23] Robinson MA, Donnelly CJ, Tsao J, Vanrenterghem J. Impact of knee modeling approach on indicators and classification of anterior cruciate ligament injury risk. Medicine and science in sports and exercise. 2014;46:1269-76. 
[24] Serrien B, Clijsen R, Blondeel J, Goossens M, Baeyens JP. Differences in ball speed and three-dimensional kinematics between male and female handball players during a standing throw with run-up. BMC sports science, medicine and rehabilitation. 2015;7:27.

[25] Pataky TC. One-dimensional statistical parametric mapping in Python. Comput Methods Biomech Biomed Engin. 2012;15:295-301.

[26] Friston KJ, Ashburner JT, Kiebel SJ, Nichols TE, Penny WD. Statistical Parametric Mapping: the Analysis of Functional Brain Images. London: Elsevier/Academic Press; 2007.

[27] Adler RJ, Taylor JE. Random fields and geometry New York: Spring-Verlag; 2007.

[28] Kal EC, van der Kamp J, Houdijk H, Groet E, van Bennekom CA, Scherder EJ. Stay Focused! The Effects of Internal and External Focus of Attention on Movement Automaticity in Patients with Stroke. PloS one. 2015;10:e0136917. [29] Carson HJ, Collins D. The fourth dimension: A motoric perspective on the anxiety-performance relationship. Int Rev Sport Exer P. 2015;9:1-21.

[30] Lee MD, Wagenmakers EJ. Bayesian Cognitive Modeling: A Practical Course. Cambridge: Cambridge University Press; 2014. 


\section{FIGURE CAPTIONS}

Fig. 1. Virtual environment.

Fig. 2. Means and coefficients of variation for step length and step width.

The first step of each perturbed heel strike was included for this graph. The big dots represent the means per condition and the small dots represent the means for each participant in each condition. Panel A shows the average step length and step width and panel $B$ shows the $C V s$. For both the means and $C V s$ no significant difference was found between internal and external focus or between fallers and non-fallers.

\section{Fig. 3. Example data of representative participant.}

The walking perturbations consist of a unilateral treadmill deceleration of the splitbelt treadmill on the participant's dominant leg side. For each perturbation the treadmill deceleration starts at toe off when there is no more contact with the dominant leg side of the treadmill. At the next heel strike the treadmill velocity on that side is $0 \mathrm{~m} / \mathrm{s}$ and starts accelerating again. Panel $A$ shows the perceived speed of the perturbed side of the treadmill. The perturbed heel strikes occur at 0 seconds. Panel B shows the earth-vertical position of the participant's COM. The red and blue lines show the mean responses of the participant to the perturbations in the external and internal focus conditions, respectively. The red and blue dashed lines show the unperturbed COM movement where unperturbed heel strikes also occur at 0 seconds.

\section{Fig. 4. COM velocity analysis.}

Panel A shows the Euclidean distance of the perturbed response COM velocity to the average unperturbed gait COM velocity. Data was normalised to stride percentage with 100 samples per stride. Each stride started at heel strike of the dominant leg, perturbed heel strikes occur at $0 \%$. Shaded areas indicate $95 \%$ confidence intervals. The horizontal dashed line indicates the relaxation distance of unperturbed gait. Panel $B$ is a vertically zoomed-in version of panel A to visualise the late response after $100 \%$.

Panel $C$ shows SPM graphs of internal, external and the difference between internal and external focus in blue, red and green respectively. Lines represent $\operatorname{SPM}\{t\}$ trajectories of the separate one-sample $t$-tests for external and internal data and paired 
$t$-tests for the external-internal difference. The SPM one-sample $t$-tests tested whether the internal and external time-series from panel A were different from the relaxation distance. Horizontal dash-dot lines are the thresholds of significance. Shaded areas are supra-threshold clusters that indicate the time domains with significant effects. The vertical red and blue lines indicate the stride percentage at which COM velocity ceased to be significantly different from the relaxation distance of unperturbed walking. Even though these stride percentages are 58\% apart for internal and external focus, no significant difference between internal and external focus was found for the Euclidean distances. 


\section{TABLES}

Table 1. Step width and step length statistics. For all $F$ values $\mathrm{df}_{1}=1$ and $\mathrm{df}_{\text {error }}=23$. The Bayes factor $\left(\mathrm{BF}_{10}\right)$ indicates the odds for the alternative hypothesis vs. the nullhypothesis to be true. For the $\mathrm{CV}$ variables of internal vs. external focus these odds are less than 1 to 3 . It has been recommended to label these Bayes factor values as moderate evidence for the null-hypothesis, while values between 1/3 and 1 were labelled as anecdotal evidence [30].

\begin{tabular}{lclll}
\hline & test stat & $\mathrm{p}$-value & effect size & Bayes factor $\left(\mathrm{BF}_{10}\right)$ \\
\hline Internal vs. external focus & & & & \\
Mean step length & $\mathrm{F}=1.21$ & 0.28 & $\eta^{2}=0.05$ & 0.35 \\
Mean step width & $\mathrm{F}=1.03$ & 0.32 & $\eta^{2}=0.04$ & 0.51 \\
CV Step length & $\mathrm{Z}=0.63$ & 0.53 & $\mathrm{r}=0.13$ & $\mathbf{0 . 2 4}$ \\
CV Step width & $\mathrm{Z}=0.79$ & 0.43 & $\mathrm{r}=0.16$ & $\mathbf{0 . 2 8}$ \\
\hline Fallers (n=8) vs. non-fallers $(\mathbf{n}=\mathbf{1 7})$ & & & & \\
Mean step length & $\mathrm{F}=0.23$ & 0.63 & $\eta^{2}=0.01$ & 0.42 \\
Mean step width & $\mathrm{F}=0.31$ & 0.58 & $\eta^{2}=0.01$ & 0.43 \\
CV Step length & $\mathrm{U}=59.0$ & 0.60 & $\mathrm{r}=0.10$ & 0.58 \\
CV Step width & $\mathrm{U}=66.0$ & 0.91 & $\mathrm{r}=0.02$ & 0.39 \\
\hline
\end{tabular}




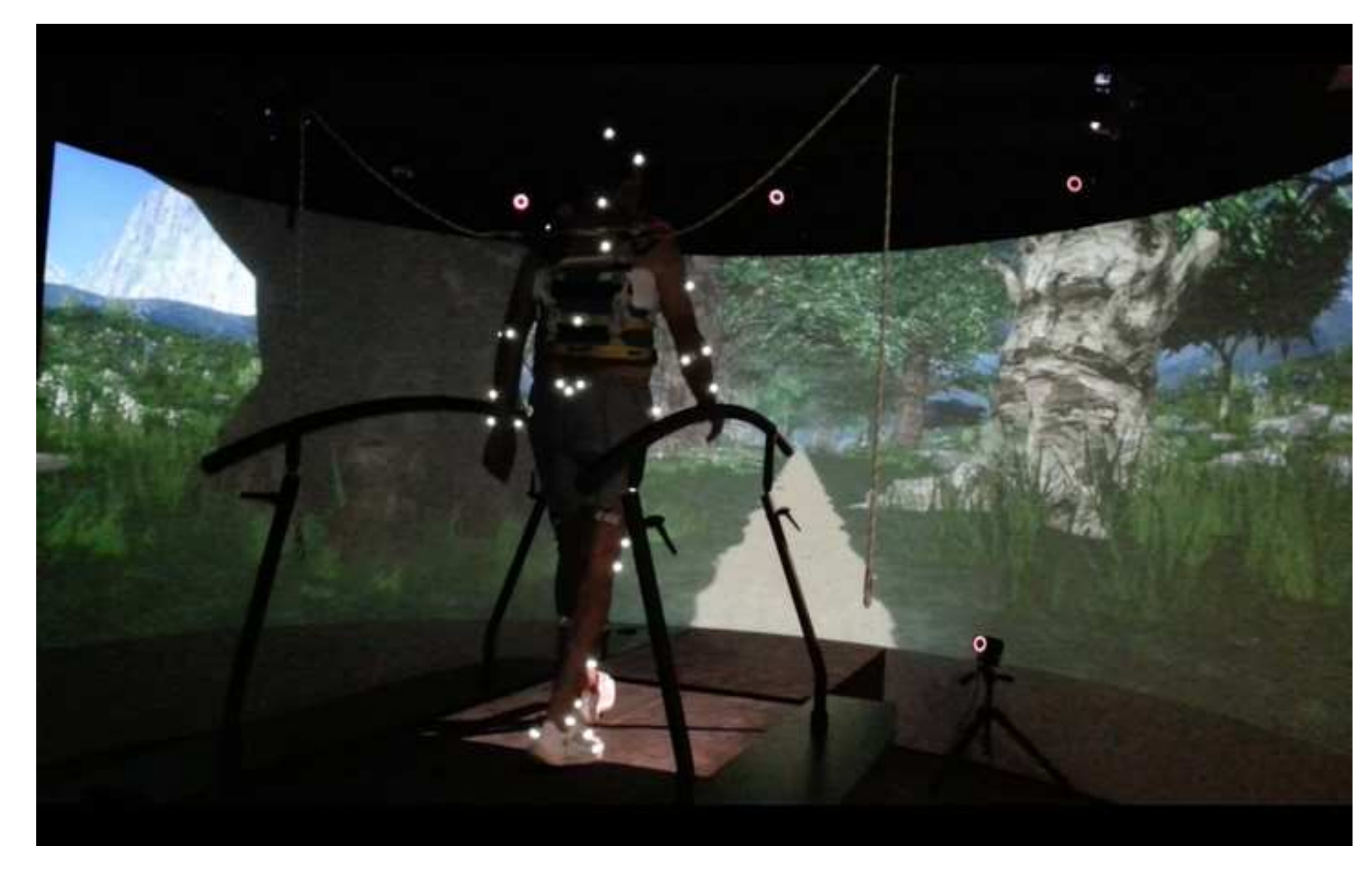

Click here to download high resolution image 

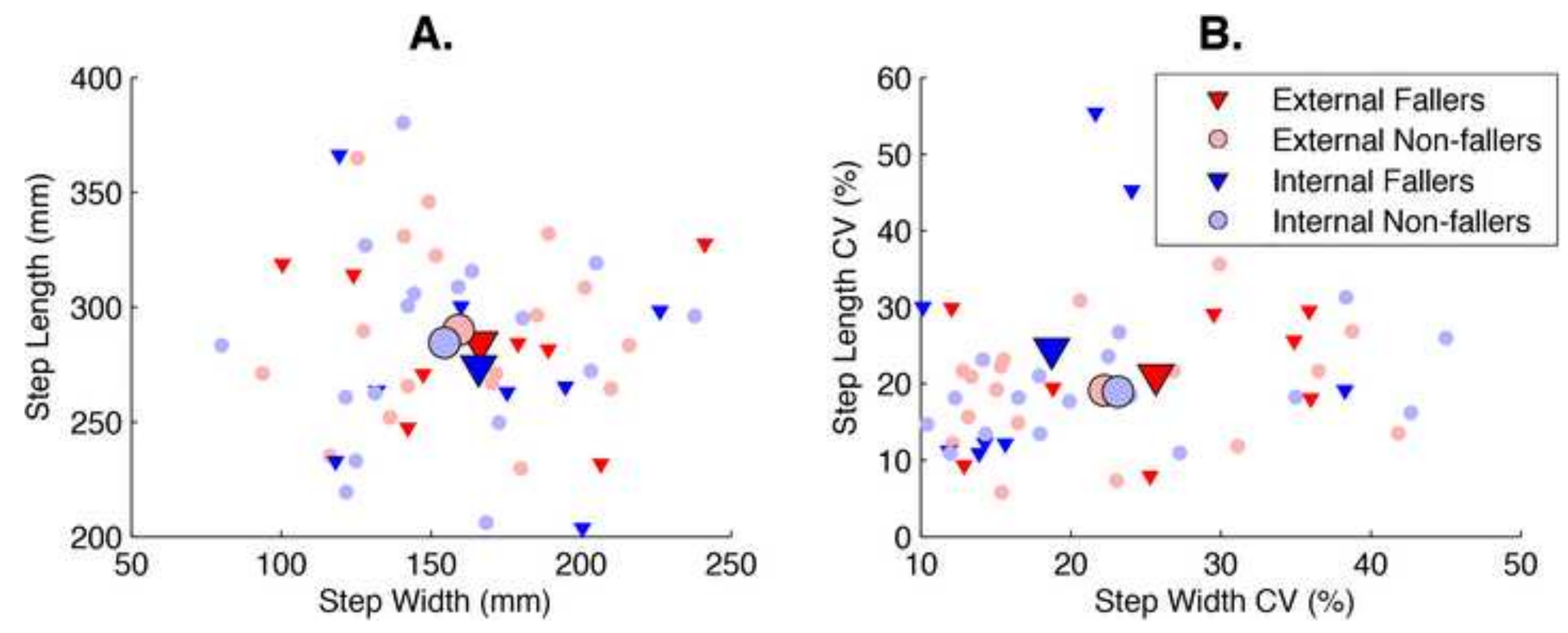


\section{A. Perceived treadmill speed}

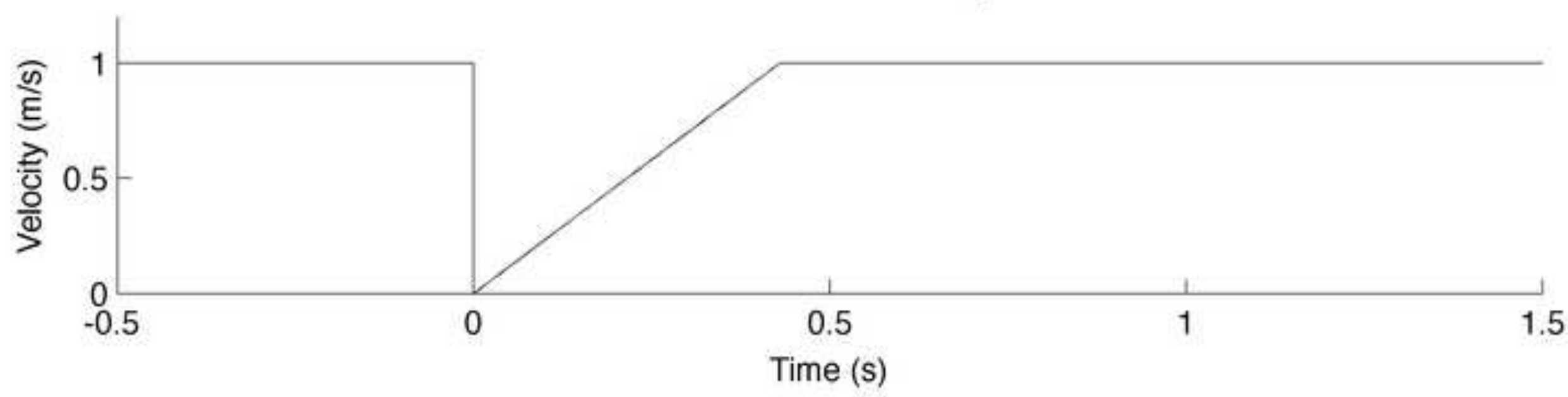

B. Earth-vertical COM position

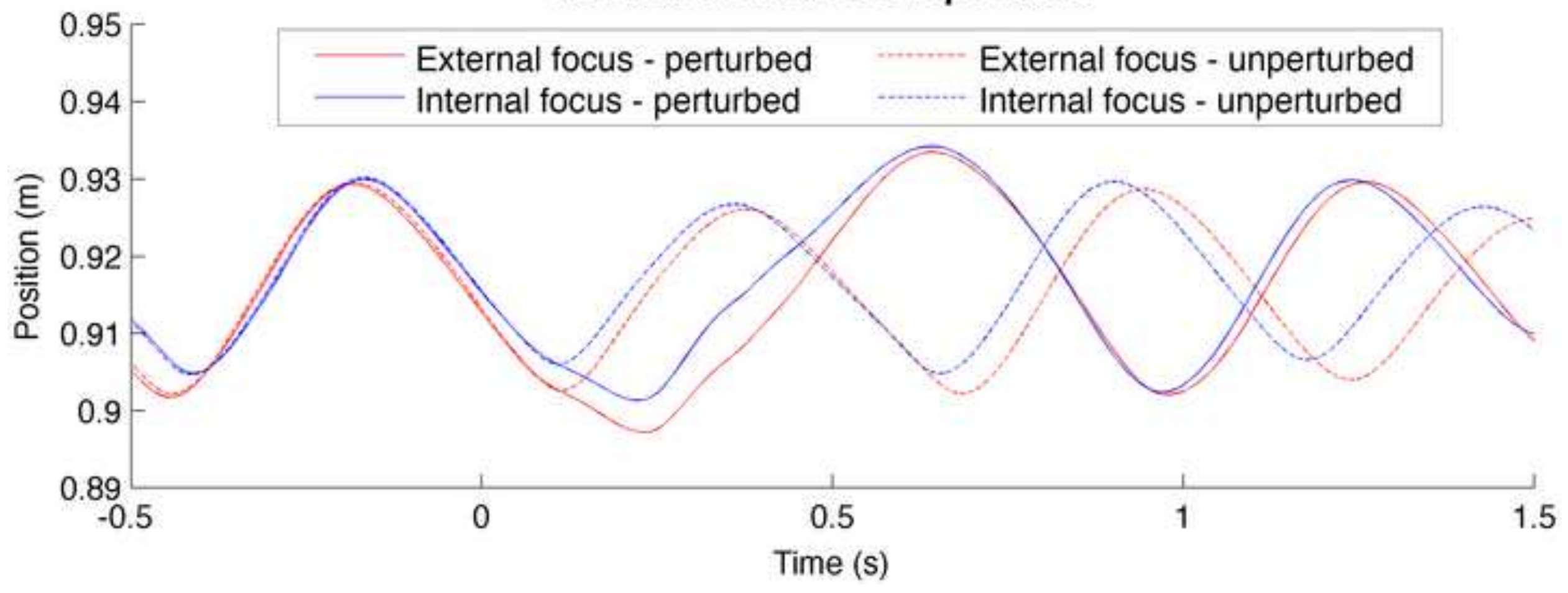



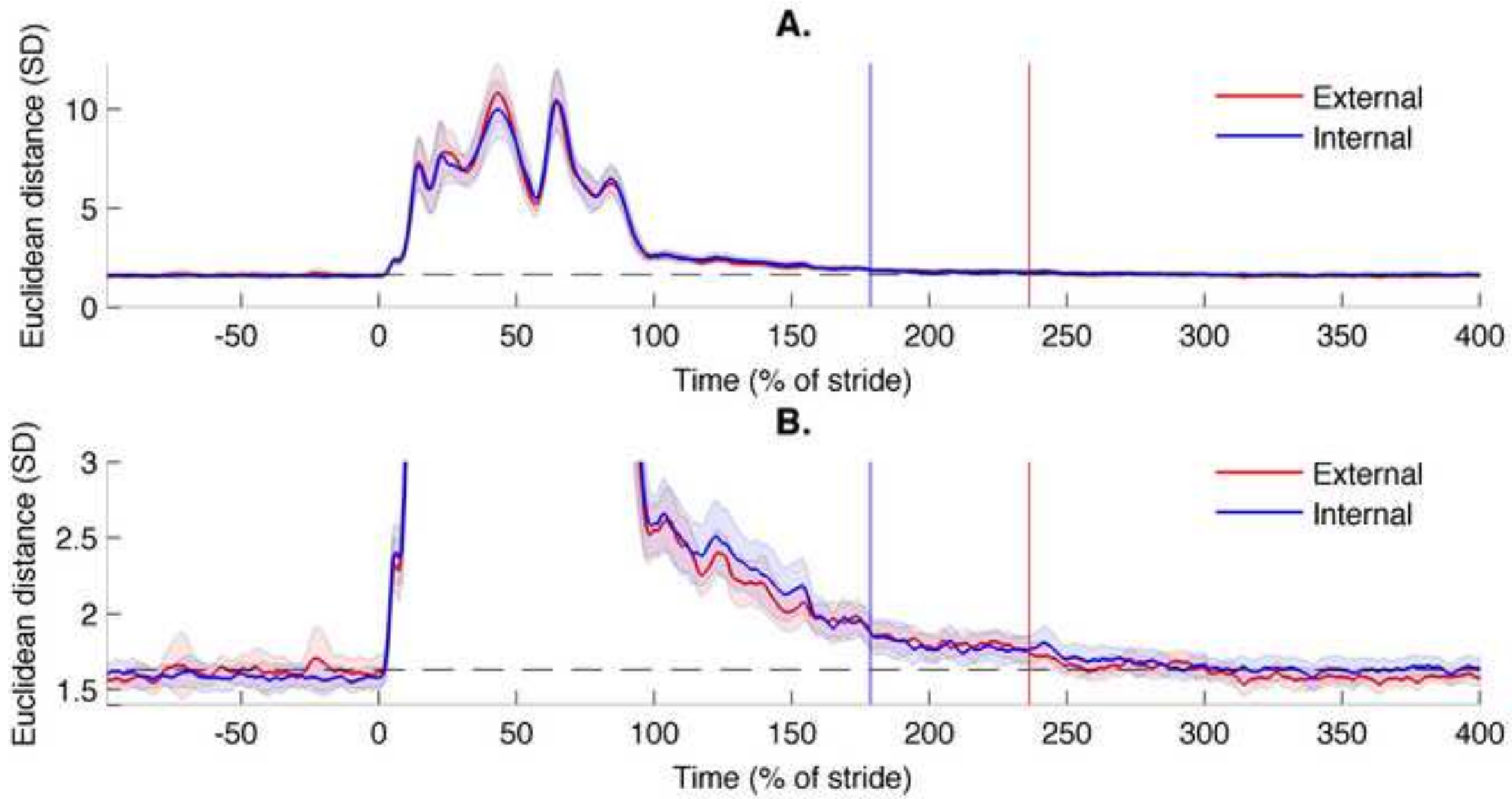

C.

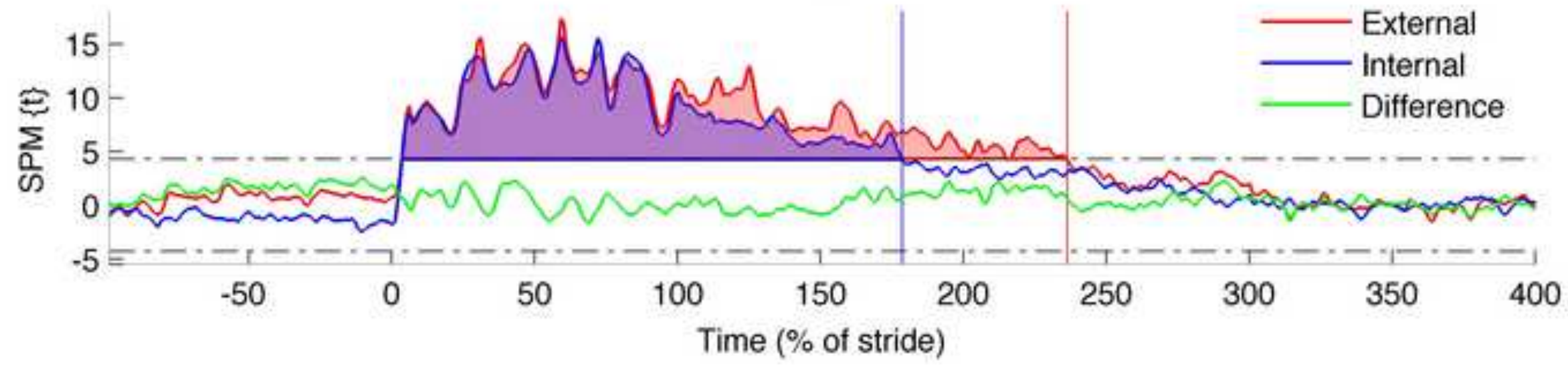


Consort 2010 Flow diagram
Click here to download 8 S

Click here to download 8. Supplementary Material: CONSORT 2010 Flow Diagram.doc

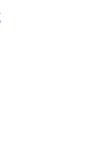

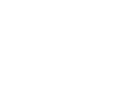

(n)

(1)

(1) $x^{2}$ $x^{2}$ $x^{2}$ . .

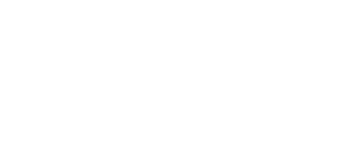
. . . . . . . . . .

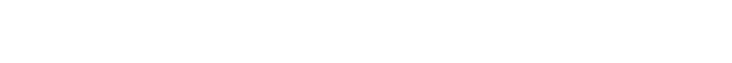
. .
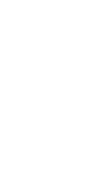

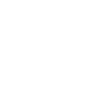
(1) $\sqrt{2}$ (1) (1)

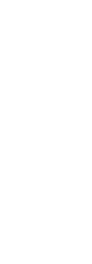

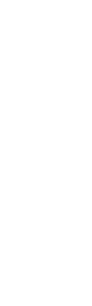

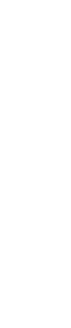

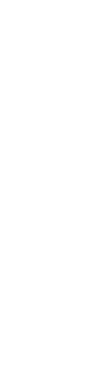
. (1) . . . (2n) 


\section{Highlights}

- Fall risk in elderly is associated with attentional focus and fall history.

- The effect of attentional focus on the stability of treadmill walking was examined.

- Internal and external focus of attention conditions were compared.

- Elderly walking stability was assessed using mechanical perturbations.

- No effects of attentional focus and fall history on walking stability were evident. 